\title{
Mil edificios, procesos maquínicos en estrategias proyectuales complejas
}

\author{
One thousand buildings, machinic processes in complex project strategies \\ Santiago Miret \\ Universidad Torcuato Di Tella, Escuela de Arquitectura y Estudios Urbanos, Argentina. \\ smiret@gmail.com
}

\begin{abstract}
This Paper refers to an ongoing Postgraduate Master thesis that is in the search to criticize the habitat devices in the framework of the collective housing project. The habitat devices are frozen configurations that hinders the processes of design in the discipline and conspires with the idea of innovation. This paper proposes new ways and models for the generative processes in Architecture. In this sense, six "disruptions" are deployed in order to attack the primary problems in collective housing projects generative processes.
\end{abstract}

Keywords: Arquitectura; procesos; proyecto; vivienda; complejidad.

\section{Introducción}

Michel Foucault nunca redactó la definición exacta de dispositivo, sin embargo, a través de entrevistas y comentarios de otros filósofos, se puede reconstruir el concepto del dispositivo. Los dispositivos refieren a manipulaciones de fuerza, inscritas en un juego de poder. Es decir, el dispositivo es una entidad capaz de manipular fuerzas $y$, al estar inmerso en relaciones de poder (o, también, surgir de ellas), resulta un arma poderosa ya sea para dirigir fuerzas en una dirección concreta, bloquearlas, estabilizarlas o utilizarlas con fines indeterminados. Por lo pronto, entonces, los dispositivos operan dentro de campos de fuerzas y relaciones y tienen el poder de alterarlos. El hecho de que los dispositivos emerjan de una condición de poder, conecta a los mismos con su genealogía de forma directa. Los dispositivos son producto de un acontecimiento cultural al cual están atados, implicando una inseparabilidad del dispositivo para con su tiempo e incluso el espíritu de su tiempo. En una entrevista de 1977, Foucault se refiere al dispositivo diciendo:

"Por dispositivo entiendo una especie, digamos, de formación que en un momento histórico dado tuvo como función mayor la de responder a una urgencia." (Foucault, 1984).

Foucault se refiere al dispositivo en términos generales, la presente tesis se enfoca en los dispositivos del habitat, es decir, los dispositivos estrictamente arquitectónicos. Foucault deja en claro que los dispositivos a los que se refiere no son sólo discursivos, sino que además pueden resultar en instituciones o, incluso, en formaciones materiales concretas. En Arquitectura existen diversos dispositivos que podrían catalogarse en función de la aproximación al problema que se enfoque. Existen dispositivos más abstractos como, por ejemplo, la noción de tipología o los hay más específicos y concretos, como el dispositivo dormitorio.
La presente tesis ${ }^{1}$ no busca criticar los dispositivos del habitat instalados culturalmente sino los mecanismos proyectuales que los involucran (voluntaria e involuntariamente) como instrumentos de acción proyectual. Es decir, no se buscará atacar el concepto de dispositivo, sino desarticular el uso de los mismos durante los procesos proyectuales de vivienda colectiva. El dispositivo disciplinar suele utilizarse como escape rápido, como atajo a la resolución de problemas, sin presentar la posibilidad a la reflexión proyectual. Así, los procesos generativos de proyecto se ven falseados y estancos a la hora de construir novedad. Lo que se buscará entonces es la constitución de nuevos modos y modelos de configuración de ciertas condiciones específicas en el caso de los conjuntos de vivienda colectiva.

En este sentido, el sujeto proyectista contemporáneo se haya sujetado por estos dispositivos del habitat de los cuales no puede escapar. El individuo proyectista se presenta escindido (en las metrópolis donde habita en la sociedad contemporánea) entre un sujeto-sujetado (condicionado a normas preestablecidas, caminos prefigurados, dispositivos del habitar instalados culturalmente, clichés) y un sujetoproductor (consciente de un Real complejo, interesado y necesitado de generar configuraciones que por dar cuenta de este real, son inéditas y rebeldes a la rutina).

Michel Foucault hace referencia a que cada episteme condiciona al sujeto ${ }^{2}$, convirtiéndolo en un sujeto-sujetado a

\footnotetext{
${ }^{1}$ Este ensayo refiere a la Tesis de Maestría en Investigación Proyectual en curso del autor denominada: "Mil Edificios, procesos maquínicos en estrategias proyectuales complejas".

${ }^{2}$ La episteme, según Foucault, refiere a aquellos marcos de saber impuestos desde el poder que inducen el comportamiento de los individuos de cada tiempo histórico. Dice Foucault: "En una cultura y en un momento dados, sólo hay siempre una episteme, que define las condiciones de posibilidad de todo saber, sea que se manifieste en una teoría o que quede silenciosamente investida en una práctica." Foucault, Michel. 1966. Les mots et les choses, une archéologie des sciences huamaines (Gallimard). Versión en español por FROST Elsa Cecilia. 1968. Las Palabras y las Cosas, una
} 
los dispositivos de poder, disminuyendo entonces la posibilidad de que el mismo desarrolle una vida auténtica. En este sentido el término tiene directa relación con lo que Martin Heidegger llama "Existencia Inauténtica", esto involucra a un sujeto que vive en "estado de interpretado". En este estado, el sujeto es incapaz de reflexionar de cara a su propia finitud y se ve restringido por condicionantes que le son externos volviéndolo un sujeto que en lugar de pensar "es pensado". Podemos hipotetizar siguiendo a Foucault que en Arquitectura el sujeto-sujetado no proyecta, sino que "es proyectado".

El problema yace en no poder dar cuenta, por parte del individuo proyectista, de esta escisión procedimental y operar, en consecuencia, en la creencia de que se están evitando aquellos lugares comunes donde el sujeto-sujetado se siente cómodo y es pensado. Es decir, el problema no radica en la escisión del individuo, sino en la creencia de que no está sujetado, o en el desconocimiento de la misma. En este sentido, el individuo opera desde el sujeto-sujetado sin dar cuenta de esta sujeción, depositando la fe del desarrollo del proyecto en su capacidad de engendrar ideas creativas, geniales u ocurrencias que emergen de la pura subjetividad, sin arraigo social ni histórico, al igual que las hipótesis que de las mismas derivan.

La tesis, entonces, no buscará liberar al sujeto de su condición de "ser pensado" sino al proyecto de su condición de "sujetado" a los condicionantes del sujeto. Específicamente en el caso de los proyectos de vivienda colectiva, los cuales caen presa de dispositivos del habitat y configuraciones predeterminadas constantemente.

Como el problema identificado son los dispositivos instalados culturalmente de la vivienda colectiva y cada uno de ellos representa una constelación de posibilidades, las cuales contienen en sí mismas los componentes (Utilitas, Firmitas, Venustas), dimensiones (teoría, metodología, técnica), contexto (disciplinar, trans-disciplinar), fines (externos, internos y mixtos) y campos (formación, investigación y profesión) que hacen a la ontología de la Arquitectura ${ }^{3}$, se los considerará multiplicidades ${ }^{4}$. En este sentido el concepto de multiplicidad hace referencia a que cada dispositivo es uno y muchos a la vez, son complejos, cambian pero son estables, se ajustan pero no tanto como para ser desconfigurados. Es por esto que cada disrupción puede ser abordada independientemente de la otra y se puede saltar de una a otra sin ningún criterio, pues cada una se ocupará en liberar al proyecto de vivienda colectiva de un dispositivo arquitectónico específico.

arqueología de las ciencias humanas (Buenos Aires: Siglo XXI Editores)

${ }^{3} \mathrm{Y}$ que constituyen las variables y sus indicadores según la epistemología de la Investigación Proyectual desarrollada por el $\mathrm{Dr}$ Arq Jorge A. Sarquis y en la cual se basa la maestría en la que se enmarca la tesis a la cual referencia este artículo.

${ }^{4}$ El concepto de multiplicidad es extraído de Gilles Deleuze quien lo explicita en su libro Deleuze, Gilles y GUATTARI, Félix. 1980. Mil plateaux, Capitalisme et schizophrénie (París: Les Editions de Minuit) Versión en español por José Vázquez Pérez y Umbelina Larraceleta, Mil Mesetas, Capitalismo y esquizofrenia (Buenos Aires: Pre-textos).
Al final, espero pueda reconstruirse (y esta es mi hipótesis) un episodio de cristalización del proyecto arquitectónico en donde cada disrupción (las cuales a la vez representan hipótesis pre-fácticas) pueda ser puesta a prueba y testeada en función de hipótesis post-fácticas. Es decir, el proceso proyectual es la tesis y el proyecto de arquitectura es la hipótesis.

\section{Asunto, tema, finalidad}

El tema de la vivienda en Arquitectura ha tomado gran relevancia en los últimos tiempos. Ya sea por problemas de espacio físico en las grandes metrópolis o por carencia en las regiones en vías de desarrollo (léase Latinoamérica, África, Asia y Oceanía). En estas regiones el problema ha alcanzado el carácter de emergencia habitacional. La demanda por la vivienda crece día a día y las respuestas que se dan por parte de los gobiernos suelen ser precarias y anticuadas. Vastas regiones del globo se pueblan de viviendas unifamiliares sin un planteo urbano, con escaso y a veces nulo equipamiento. Otra respuesta es la generación de edificios de vivienda colectiva exactamente iguales replicados ad-infinitum en planicies con escaso planteo organizativo. La agenda disciplinar hoy exige un desarrollo del proyecto de vivienda colectiva entendido como "contemporáneo y situado". Dando cuenta de la necesidad de ocupar el espacio físico de las ciudades de modo inteligente ${ }^{5}$, sustentable y comprometido con la historia disciplinar. Entonces, se han identificado dos problemas en relación a la vivienda colectiva contemporánea:

1. La emergencia habitacional en las regiones de países en vías de desarrollo. La estrepitosa parafernalia del llamado "Star System" y los grandilocuentes concursos internacionales de Arquitectura parecen haber olvidado la creciente necesidad de las regiones más afectadas del planeta. El crecimiento de los asentamientos precarios en ciudades como Buenos Aires, Rio de Janeiro, Nueva Delhi, Johannesburgo, entre muchas otras de los últimos años dan cuenta de este flagelo.

2. La falta de actualización de los programas de vivienda. Tanto en su carácter material (resoluciones técnicas) como en su modo de aparecer proyectual (configuraciones anticuadas, repetitivas, que no dan respuesta a los nuevos grupos familiares).

En este sentido considero fundamental operar desde el proceso de proyecto, ofreciendo alternativas a los modos de acción contemporáneos que parecen desembocar una y otra vez en configuraciones repetitivas y carentes de propuesta. Dormitorios, comedores, baños y cocinas (estereotipos diseñados como ámbitos culturalizados) parecen ser la única respuesta a los sub-espacios de la vivienda a nivel internacional. La híper-globalización ha generado un vasto horizonte de homogeneidad. Las nuevas y emergentes herramientas digitales son utilizadas como potenciadoras de la visualidad y los tiempos de producción, mientras que los

\footnotetext{
${ }^{5}$ En el sentido de que se debe hacer uso de la superficie y el máximo volumen edificable de una manera eficiente sin desperdiciar espacio o posibilidades de crecimiento.
} 
métodos siguen siendo los mismos de hace un siglo en algunos casos y en otros aún más antiguos.

La vivienda colectiva se instala definitivamente como tema de relevancia disciplinar durante la segunda post-guerra vehiculizado por las exploraciones de Moisei Ginzburg (18421946) y Le Corbusier (1887-1965), entre otros. Al día de hoy se han instalado con tanta fuerza aquellas ideas (relacionadas con un saber universal, un enamoramiento tecnológico, un paradigma de la simplicidad según Edgar Morin $^{6}$ ) que el sujeto proyectista contemporáneo no puede desligarse por completo de ellas.

El modo de operar en Arquitectura, en el marco de lo que implica una contemporaneidad compleja, dadas las condiciones de un sujeto tensionado entre fuerzas conservadoras por un lado y propositivas por el otro, será por medio de una entidad maquínica proyectual que produzca corrimientos, desfasajes y disrupciones entre el sujeto proyectista (Arquitecto) y el objeto (proyecto de Arquitectura), propiciando instancias de autonomía procesual por parte de dicha entidad potenciadas por el uso de metodologías de proyecto digitales generativas que permitan la manipulación de grandes cantidades de información.

\section{Enunciado Proyectual}

Cada una de las tesis se enfoca en problemas específicos del proceder proyectual de la vivienda colectiva desde la generalidad a la especificidad.

1. La concepción de volumen legal como límite.

>relación con la LEGALIDAD

$>$ instrumento CAMPO

2. El referente histórico como dispositivo.

>relación con la HISTORICIDAD

> instrumento ALGORITMO

3. La comodidad del índice estático.

>relación con la REFERENCIALIDAD

> instrumento TOPOLOGÍA

4. La limitación de la noción de publicidad.

>relación con la SOCIABILIDAD

> instrumento NO-LINEALIDAD

\footnotetext{
${ }^{6}$ Ver: MORIN, Edgar. 1994. Introducción al pensamiento complejo (Barcelona: Gedisa). Morin llama paradigma de la simplicidad a aquel pensamiento atado a la idea cartesiana de mundo escindido entre materia y pensamiento. El modo de entender el real atado a creencias de Verdad y progreso.
}

5. El miedo por lo heterogéneo.

$$
\text { >relación con la COMPLEJIDAD }
$$

$$
\text { > instrumento PROTOCOLO }
$$

6. La asociación de la vivienda colectiva con la familia.

$$
\begin{aligned}
& \text { >relación con la INDIVIDUALIDAD } \\
& >\text { instrumento GRADIENTE }
\end{aligned}
$$

\section{No Fondo/Figura Sino Continuo/Emergente}

"Una condición de campo seria cualquier matriz formal o espacial con la capacidad de unificar distintos elementos siempre que respete la identidad de cada uno. Las configuraciones de campo son conjuntos ligeramente vinculados caracterizados por su porosidad y conexión local. Las regulaciones internas de las partes son decisivas, sobre todo si su forma tiene alta fluidez."

Allen, Stan. 2009. "Del objeto al campo" en Architectural Design (67), 24-31.

El concepto de fondo/figura es un dispositivo del hábitat de la vivienda colectiva que condiciona al individuo proyectista. No se trabajará insertando una figura sobre un fondo, sino en la construcción de un continuo emergente de las condiciones de materia latentes del contexto inmediato.

Stan Allen, en su famoso ensayo sobre el campo, pone de manifiesto la necesidad de la disciplina de pensar las edificaciones como continuos emergentes de las condiciones contextuales e internas al proyecto. Esto quiere decir que un objeto posado sobre el paisaje es siempre un problema. La idea de tabula rasa implementada y desarrollada por el llamado Movimiento Moderno tiene su nacimiento en el plano de la ciudad de Roma dibujado por Giambattista Nolli en $1748^{7}$. El cual construye a priori una idea de objeto sobre plano. Sin construir relaciones entre objetos más que la traza preexistente y las condiciones propias de un crecimiento de ciudad espontáneo. Las ciudades ya sean planificadas o no que a continuación se desarrollaron siguieron esta idea de la condición de la Arquitectura como objeto posado sobre un plano homogéneo e inactivo.

Stan Allen pone de manifiesto en su artículo es que no obstante esta condición de escisión entre el objeto y su emplazamiento, la relación de continuidad siempre estuvo presente. De alguna manera siempre se ha operado desde una condición de campo. Sin embargo, estas relaciones de campo tendían a ser intuitivas, ocurrentes según se presentara el caso. Los objetos posados en el paisaje tenían relación para con las condiciones climáticas (orientación), o con el follaje circundante 0 , incluso, con arterías urbanas. Pero estas relaciones se vieron siempre ocultas en los procesos generativos de proyecto, dando por sentado su existencia de manera vaga y general.

\footnotetext{
7 Ver: Map of Rome, Nolli, Giambattista, 1748. Fuente: Earth Sciences \& Map Library-University of California, Berkeley.
} 
Esta disrupción propone reconstruir las relaciones de campo entre los elementos que operan en el emplazamiento del proyecto a saber, en este caso: 1. Límites legales. 2. Adyacencias edilicias. 3. Construcciones pre-existentes. Así se buscará el desarrollo de un manto continuo que opere, no como mediador hibridizante de estas condiciones preexistentes sino como aglomerador continuo de las mismas con el objeto de hacer emerger una cuarta condición de campo que será la propositiva en relación a la condición de heterogeneidad del proyecto.

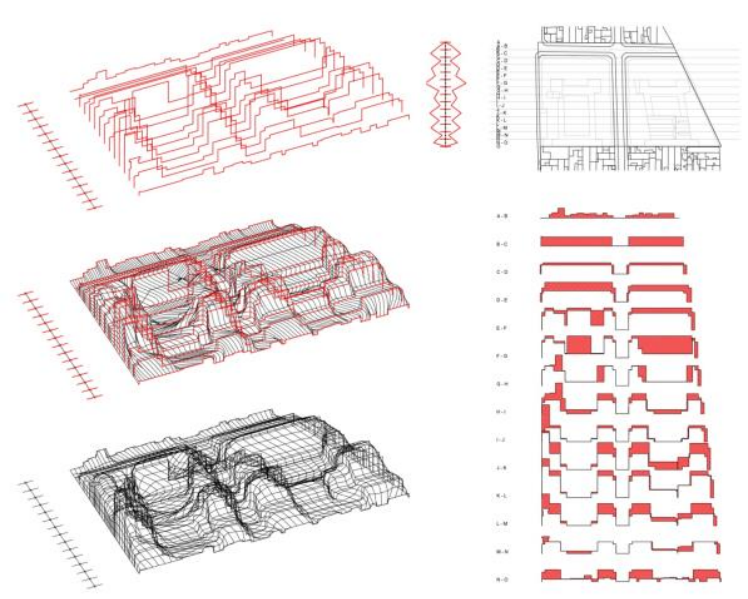

1. Construcción de la matriz. Derecha Arriba: Secciones finales. Derecha Centro: Superficie emergente. Derecha Abajo: Subdivisión del manto según un promedio de celdas de 30m2. Izquierda: Estudio de secciones generativas según ajuste. Análisis de superposición - 14 subdivisiones

Fuente: Tesis del autor.

\section{Ni Siedlung, Ni Matbuilding Sino Síntesis No-Figurativa}

"Argumentaré que la idea de Siedlung no estaba errada, sino que fue pobre 0 inadecuadamente conceptualizada, particularmente en relación al cambio de ideas en relación a lo individual y a la repetición mecánica. Es así que un regreso a las estructuras del pasado no es la solución a la forma urbana de hoy en día, pero quizá una reconsideración puede proveer alguna solución."

Eisenman, Peter. 1991. "Folding in time, the singularity of Rebstock"

La vivienda colectiva tradicionalmente se asocia con pabellones de alta densidad o con grandes extensiones de baja densidad, esto representa un dispositivo. Se buscará la síntesis de ambas condiciones de pre-figuración con el objeto de obtener un sistema de densidad variable de condiciones indeterminadas.

A la hora de consolidar proyectos de vivienda colectiva, existen dos tipologías edilicias que emergen como condición determinante. Por un lado la idea de alta densidad y aprovechamiento del suelo mínimo asociada al pabellón de viviendas (en Alemán Siedlung) emergente como tal durante el período de entreguerras en Alemania y asociado al concepto de espacio mínimo. Durante el período de recuperación y reconstrucción de Europa, la necesidad de construir alta densidad en poco espacio y de forma barata fue lo que impulsó al desarrollo de grandes conjuntos de vivienda colectiva compuestos por pabellones de viviendas independientes. Los cuales constituían un campo modulado de estructuras autónomas e idénticamente repetidas. Con el tiempo esta idea fue transpolada a otras condiciones temporales y contextuales constituyéndose como un dispositivo ineludible de la vivienda colectiva en la disciplina. Tal es así que en muchos territorios estos modelos han demostrado ser erróneos, ya sea por la inabarcabilidad de regulación en relación a consorcios de gran escala o por la falta de relación con las necesidades intrínsecas del contexto en el que se implantaron. Casos testigo en nuestra región son el conjunto urbano Soldati proyectado por el grupo Staff en 1979 o el conjunto urbano Comandante Piedrabuena proyectado por Manteola, Sanchez Gómez, Santos, Solsona Arquitectos en 1981.

El otro modelo de vivienda colectiva al que se hace referencia es el de Mat-Building u, opuestamente al anterior, la idea de población superficial de baja densidad. Así, lo que predomina es la repetición en serie de viviendas unifamiliares o conjuntos de dúplex y triplex, los cuales se emplazan sobre el territorio ad-infinitum como alfombras poblacionales extensísimas. Esta estrategia no sólo deviene en problemas infraestructurales en relación a problemas en el suministro de agua potable, cloacas, gas y electricidad, sino que además contribuye a la generación de grandes fragmentos urbanos aislados sin acceso franco a instituciones educativas, áreas de esparcimiento y transporte.

La presente disrupción busca consolidar aspectos de relación entre estas dos tipologías, focalizando más específicamente en las condiciones de iluminación y ventilación asociadas a la profundidad de planta de estas tipologías, consolidando un conglomerado continuo en donde no sea precisamente distinguible la condición de pabellón ni la de Mat-Building como tales.
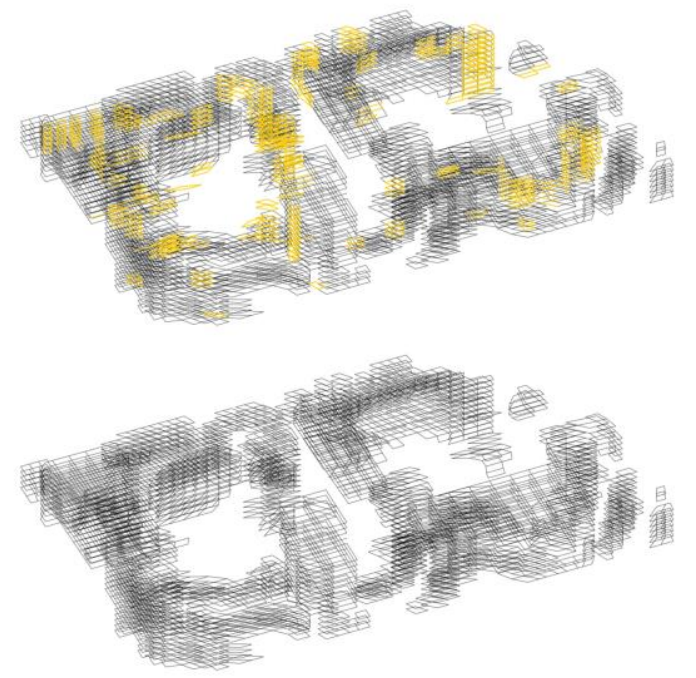

2. 6ta etapa de esponjamiento $(<20 \mathrm{~m} 2)$. Esponjamiento de celdas según metros cuadrados. 
Arriba: Axonometría de la estratifiación esponjada ajustada y celdas menores a $20 \mathrm{~m} 2$ seleccionadas.

Abajo: Axonometría del esponjamiento resultante de eliminar las celdas menores a $20 \mathrm{~m} 2$.

Fuente: Tesis del autor.

\section{No Tipología Sino Topología}

"El diagrama es una matriz invisible, una serie de instrucciones, que subyace - y más importante, organiza - la expresión de características en cualquier constructo material. El diagrama es el reservorio de potencial que yace a la vez activo y en reserva en un objeto o un ambiente (o en cada agregado o sección de éstos)."

Kwinter, Sanford. 2006. "The Judo of Cold Combustion" en REISER, Jesse. 2006. Atlas of Novel Tectonics (Nueva York: Princeton Architectural Press).

El uso de tipologías en los proyectos de vivienda colectiva responde a una comodidad por parte del individuo proyectista, sin dar lugar a configuraciones de unidades de vivienda más allá de las pre-figuradas por el mismo. El concepto de topología pretende distorsionar la idea de tipología y, por medio de la proliferación informacional, generar geometrías inesperadas.

La noción de tipología en relación a las unidades de vivienda colectiva ha llegado a constituirse como un dispositivo que evita pensar el modo de apropiación de las unidades. El proyecto de vivienda colectiva se ve atada a la condición tipológica de las unidades habitativas que la componen. Así, los conjuntos de vivienda colectiva suelen buscar la diferencia en condiciones de fachada, uso de materiales, implantación, forma general, etc. Pero no se ahonda en la reconfiguración o innovación en cómo las unidades responden a los modos de habitar emergentes en la actualidad.

Así como la idea de familia se encuentra en crisis tanto a nivel disciplinar como cultural (ver Disrupción 06, no familia, sino individuo) el modo en que las familias se apropian de las unidades de vivienda también. Las tipologías de vivienda suelen variar según cantidad de ambientes, cocina integrada o separada, con o sin balcón y demás condiciones generales. Tanto se ha hecho hincapié en este tipo de estructuraciones que ya no es necesario revisitar los modos de habitar a la hora de proyectar estas unidades. Simplemente basta con recurrir al catálogo de tipologías de vivienda que la misma disciplina se ha encargado de generar para "elegir" la que mejor sirve a nuestros propósitos formales. De este modo se pueden encontrar en la disciplina dos maneras de operar tipológicamente. El primero es cuando la tipología es la que construye la totalidad. En este caso, las unidades tipológicas dan forma al conjunto por medio de un apilamiento repetitivo y modular como en el caso del conjunto de viviendas Habitat 67 proyectado por Moshe Safdie en 1967. El segundo modo de operar tipológicamente se refiere al modelo por el cual la tipología de vivienda se adapta a una forma del conjunto. En este caso la tipología debe responder a un a priori formal constituido por la totalidad. Casos testigo son algunos proyectos del grupo MVRDV como, por ejemplo, el complejo Mirador en Madrid, España o el Silodam en Países Bajos.
La presente disrupción desarrollará un modelo generativo de unidades asociado a una acción global sobre el conjunto, la cual proporcionará herramientas geométricas para la consolidación de unidades topológicas y así ponerlas en relación gracias a la identificación de cuatro tipos de sectores: 1. Sector íntimo. 2. Sector de intercambio. 3. Sector de conexión. 4. Sector emergente (por superposición). De este modo, se podrá construir un catálogo topológico el cual emergerá de las condiciones intrínsecas al proyecto en donde la condición de tipología y conjunto se vea como un continuo indiferenciable. Las topologías emergentes de este proceso darán cuenta de modos de habitar diversos pudiendo consolidarse unidades habitativas heterogéneas y dando la posibilidad de ser habitadas por grupos familiares de amplia diversidad en relación a sus intereses.

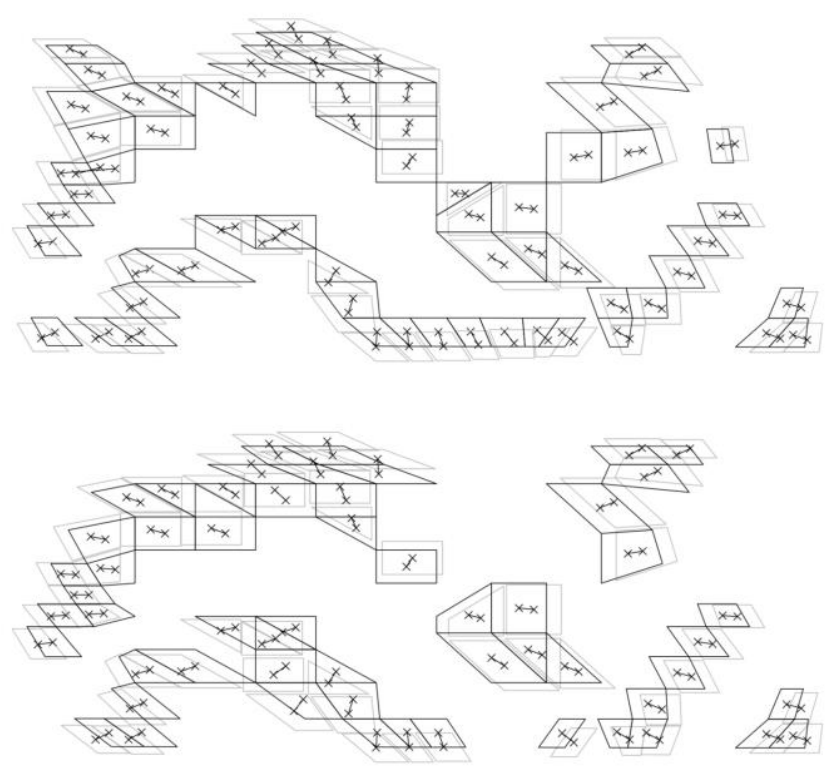

3. Desfase de celdas. Generación del desfasaje de celdas según instancias de intimidad - conector - intercambio (Detalle de un sub-conglomerado).

Arriba: Nivel -5

Abajo: Nivel -4

Fuente: Tesis del autor.

\section{No Patio, Tampoco Patios Sino Gradientes De Publicidad}

"El término 'no-lineal' es utilizado para referirse a ecuaciones en donde la información de entrada (input), no tiene siempre un correlato de causa y efecto con la información de salida (output)“

Carpo, Mario. 2013. The Digital Turn in Architecture 1992 2012 (Londres: John Wiley \& Sons).

La generación de guetos en los conjuntos de vivienda colectiva representa un problema asociado a la poca variabilidad de condiciones de sus instancias interiores. Los gradientes de privacidad externa, permiten una apropiación más acorde a la complejidad de diferenciación que implica la 
vida íntima, la sociabilidad interna y la conectividad con un exterior.

Las instancias de espacio de intercambio público en un conjunto de vivienda colectiva son fundamentales. La idea de vivir colectivamente se funda en la noción de compartir el espacio y, a la vez, aprender de esta actividad en razón de vivir socialmente. Sin embargo, esta condición de sociabilidad en los conjuntos de vivienda colectiva suele ser relegada a espacios de no más de tres gradientes de privacidad, de los cuales la mayoría no consolidan instancias de lugares para la vida en comunidad. Estos ámbitos pueden identificarse como: 1. Palieres. 2. Salones de Usos Múltiples. 3. Patios comunes. En estos lugares no se consolidan gradientes de publicidad, ya que en los palieres no existen instancias de intercambio o lugares donde desarrollar actividades. En los Salones de Usos Múltiples las actividades se hayan acotadas a un grupo pre-establecido de individuos sin interés de sociabilización con el resto. Finalmente, los patios comunes son los más interesantes en términos de sociabilización. Sin embargo, no constituyen áreas de intercambio diferenciadas y su modo de apropiación suele ser espontáneo 0 sin ningún tipo de propuesta arquitectónica.

Esta disrupción interpretará los tipos de unidades habitativas que rodean los espacios comunes con el fin de identificar qué tipo de condición de exterioridad en relación a los tipos de individuos que habitan esas unidades se corresponde con ese patio. De esta manera proporcionará la instancia de diferenciación entre gradientes de publicidad, en principio, indeterminados. Dando lugar a la generación de patios compartidos entre más o menos cantidad de individuos con el fin de proporcionar instancias de intercambio social diferenciadas a lo largo de todo el conjunto.

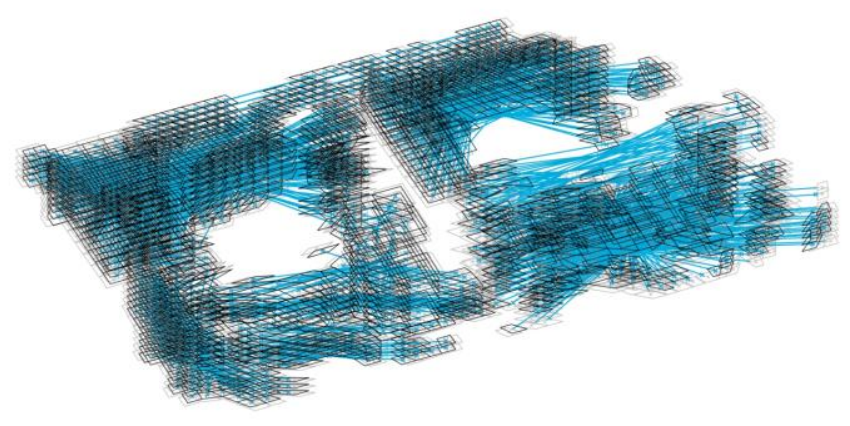

4. Asociación de unidades habitativas al espacio público. Identificación de distancias de proximidad.

Fuente: Tesis del autor.

\section{No Conexión De Homogeneidades Sino Cohesión De Heterogeneidades}

"Para decir que algo es un sistema, debemos poder definir claramente: 1. El comportamiento holístico al que se enfoca, 2. Las partes del objeto y las interacciones entre las partes, que producen de hecho el comportamiento holístico que hemos definido y 3 . El modo en que la interacción entre las partes produce el comportamiento holístico que hemos definido".

Alexander, Christopher. 1969. Tres Aspectos de Matemática y Diseño (Barcelona: Tusquets).

El concepto de disponer de homogeneidades las cuales conectar representa un dispositivo para la facilitación de la tarea de unión. Por medio de tácticas de cohesión se afectará la forma para que, en su heterogeneidad, aparezca como un continuo. En donde cada parte contiene indicios de continuidad de la siguiente.

Los conjuntos de vivienda colectiva son homogéneos. O, mejor dicho, resultan en una conexión de homogeneidades. Tanto la homogeneización de la idea de tipología como la de pabellón o mat-building, concluyen en la necesidad de conectar por medio de pasillos, puentes o senderos una serie de homogeneidades inactivas. Esto repercute en ideas de serialización indefinida, donde la idea de infinitud juega un papel clave en su inevitable repetición modular.

En función de comprender al proyecto de vivienda colectiva como un sistema reactivo, será necesario entender a sus partes como componentes de un sistema arquitectónico, los cuales han de reaccionar en función de la variabilidad. Si en un conjunto de vivienda tradicional existen tipologías homogéneas conectadas por senderos, en un conjunto de viviendas donde las unidades habitativas son heterogéneas, este sendero ha de ser reactivo a esta heterogeneidad, no sólo siendo modificado el mismo, sino además modificando las unidades a las que sirve. De este modo ya no se trata de espacio sirviente y espacio servido, sino que ambos reaccionan en función de una condición relacional que es continuamente heterogénea.

Esta disrupción generará conexiones diferenciadas según nivel conectando instancias de heterogeneidad entre unidades a la vez que modifica a su paso las unidades a las que conecta. Constituyendo instancias de conectividad tanto horizontales como verticales y concatenando condiciones heterogéneas diversas a diferentes niveles. 


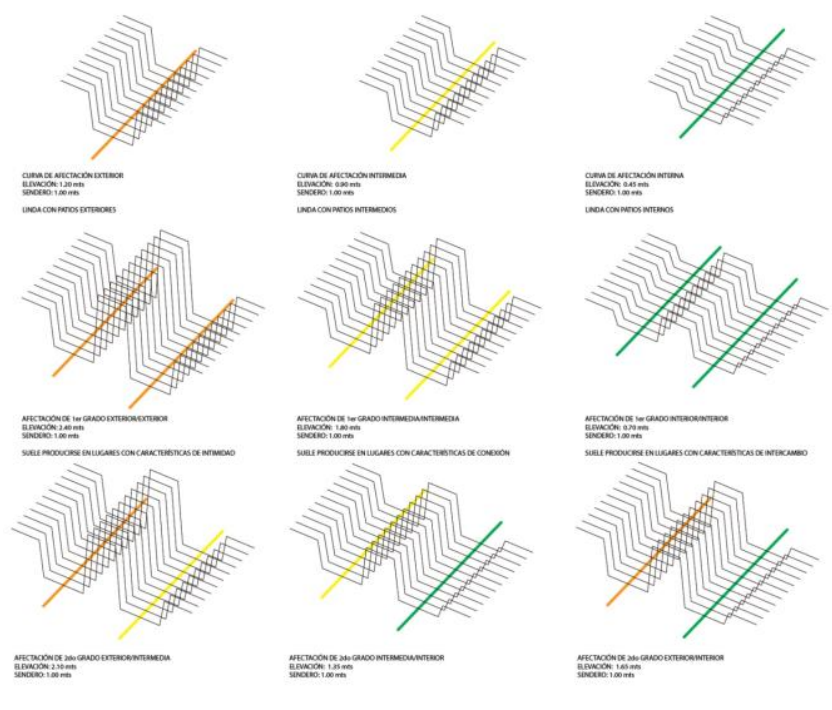

5. Catálogo genérico de distorsión de las superficies habitativas según tres tipos de relación de adyacencia.

A. Patios exteriores / B. Patios intermedios / C. Patios internos.

Fuente: Tesis del autor.

\section{No Familia Sino Individuo}

"From then on, we not only would have a single mind and body, but many minds - conscious and unconscious - and many bodies, all incoherent, all incommensurate, yet somehow converging into a singular manifold."

Kipnis, Jeffrey. 2013. A question of qualities (Massachusetts: The Mit Press).

El concepto de unidad habitativa es un dispositivo heredado de concebir a la familia (ideal) como unidad mínima del conjunto de vivienda colectiva. La posibilidad de disolución de la familia, nos permite pensar en términos del individuo y su multiplicidad como entidad autónoma y compleja en sí misma. Esto deriva en la gradación de instancias de privacidad internas del habitar.

El concepto de vivienda colectiva es uno de los más nuevos en la agenda disciplinar de la arquitectura. No fue sino hacia principios del siglo XIX que las más pujantes ciudades europeas comenzaron a poblarse de trabajadores que, escapando del hambre de las zonas rurales, recurrían a los focos de industrialización urbanos con la esperanza de una vida nueva y llena de posibilidades. Es en ese momento que la idea de vivienda en su carácter colectivo comienza a gestarse como tema de la arquitectura. Sin embargo, éste no llegará a afianzarse como tal hasta el período de entreguerras con algunas exploraciones arquitectónicas que buscaban dar respuesta a la necesidad de reconstrucción de la devastada Europa. Finalmente es el período de la segunda pos-guerra cuando la vivienda colectiva emerge, no sólo como tema disciplinar, sino también como ejemplo de la práctica arquitectónica con un sinfín de nuevas tipologías y experimentaciones formales, que ya comienzan a formar parte de su ontología.

El énfasis con el que los congresos $\mathrm{CIAM}^{8}$ principalmente y la llamada Arquitectura Moderna trataron el tema de la vivienda colectiva devino en la creencia que la idea de vivienda era esencialmente Moderna. Es así que durante ese período occidente identificó un tipo de vivienda con la idea de vivienda universal. Dejando de lado cualquier otro tipo de conceptualización posible. Es durante este período que la vivienda colectiva se instala como tema disciplinar más no de investigación, sino como respuesta a una necesidad práctica deviniendo en configuraciones (tanto espaciales, como materiales y de actividades) rígidas, estáticas, plagadas de los condicionantes propios de los Maestros Modernos, como las ideas de función, tecnología, higienismo y practicidad. Pero más importante, el concepto de vivienda se tiñó de síntomas morales y éticos los cuales marcaron no sólo al acontecer histórico sino al devenir proyectual futuro de la vivienda colectiva. Así como la vivienda emerge a principios del siglo XIX como un problema de la industrialización, hacia la segunda posguerra se consolida como la necesidad de reconstruir la idea de familia. $Y$ esta idea, al no responder a una necesidad material (como sí lo hacía el primer período) se vuelve en una carga moral de la cual la vivienda como agenciamiento disciplinar no podrá despegarse tan fácilmente.

Considero que el principal interés ha de estar situado en la posibilidad de desestructuración del concepto de "unidad habitativa", en función de constituir "multiplicidades habitativas" sin la necesidad de consolidar unidades autónomas y completas, dando lugar a la incompletud y ambigüedad que las teorías de la complejidad se han encargado de desarrollar tan minuciosamente en otras disciplinas. Sólo que esta vez el punto está situado en las actividades del habitar y cómo estas resultarán en nuevos modos de producir proyectos de vivienda, pero sobre todo nuevos modos de entender la disciplina.

\section{Evaluación}

Luego del despliegue del proceso de proyecto arribamos a un proyecto de arquitectura, el cual ha de ser puesto a prueba con miras a evaluar el grado con que las condiciones descriptas en las disrupciones precedentes se verifican en el resultado final. A partir de esto se desarrollará una conclusión en relación al problema y la hipótesis general.

Siendo el presente un trabajo en proceso, no hemos arribado aún a conclusiones definitivas. Sin embargo, se pueden dilucidar aspectos generales en relación a una evaluación de lo hecho hasta aquí. Los procesos generativos de vivienda colectiva se encuentran plagados de dispositivos. Los aquí desarrollados son sólo 6 , los cuales resultan determinantes a la hora de generar el proyecto. No obstante resultan muy generales, son ampliamente abarcativos. En este sentido, se podrían analizar separadamente en función de cuáles serían los aportes en términos de conclusiones que se espera de cada uno de ellas.

Disrupción 01. No fondo/figura sino continuo/emergente.

8 CIAM: Congresos Internacionales de Arquitectura Moderna. Llevados a cabo desde 1828 hasta 1959. 
Se busca distorsionar la idea de fondo/figura poniendo de manifiesto las condiciones relacionales pre-existentes en el campo de acción del proyecto. El objetivo es constituir un manto que accione como límite de las tácticas subsiguientes.

Disrupción 02. Ni siedlung, ni matbuilding sino síntesis nofigurativa.

Se busca operar en el intersticio entre la condición de pabellón y mat-building de viviendas. De este modo constituir un conglomerado de unidades que responden a parámetros generales de ventilación e iluminación con el fin de conjugar alta densidad y porosidades regulares en un continuo edilicio compacto.

Disrupción 03. No tipología sino topología.

Se busca una alternativa a la idea de tipología de unidad habitativa. En este sentido, se incorporan para la generación de unidades condiciones de interioridad de la vivienda como criterios de apropiación de los espacios. De este modo se genera un catálogo topológico de unidades habitativas emergentes de las condiciones generales del conjunto sin la necesidad de recurrir a tipos pre-establecidos.

Disrupción 04. No patio, tampoco patios sino gradientes de publicidad.

Se busca constituir instancias de espacios públicos diferenciados según posibilidades y oportunidades de apropiación asociadas a las unidades habitativas de las que dependen y se desprenden. Los lugares de intercambio público resultan en instancias diferenciadas de gradientes de apropiación diversos en donde grupos de diversa índole y tamaño pueden hacer uso de los mismos dependiendo de sus intereses y las condiciones sociales que los rodean.

Disrupción 05. No conexión de homogeneidades sino cohesión de heterogeneidades.

Se busca consolidar un mecanismo de conexión entre unidades que no sea homogéneo y que produzca alteraciones en las condiciones internas de las mismas. De este modo las instancias de conexión se sintetizan junto con los gradientes de publicidad (exterior) y los gradientes de intimidad (interior) consolidando un conglomerado de unidades interconectadas de modo heterogéneo.

Disrupción 06. No familia sino individuo.

Se busca una alternativa al dispositivo que implica la idea de familia a la hora de proponer conjuntos de vivienda colectiva. La disrupción plantea una posibilidad de apropiación interna de las unidades de vivienda asociada a intereses individuales que, por medio de complejas interacciones entre los mismos, se constituyen en un conglomerado habitativo diverso y compacto.

El trabajo representa una crítica propositiva a los modos tradicionalmente instalados en la disciplina de proyectar conjuntos de vivienda colectiva atacando aquellas instancias del proceso que se ven atadas a dispositivos disciplinares. En este sentido se logra construir una alternativa a los modos estancados de pensar la vivienda colectiva y se busca construir un conocimiento que sirva a futuro como manual operativo en lo que a procesos generativos de vivienda colectiva refiere.

\section{Bibliografía}

Alexander, Christopher. (1969). Tres Aspectos de Matemática y Diseño. Barcelona: Tusquets.

Allen, Stan. (2009). "Del objeto al campo" Architectural Design (67), 24-31.

Carpo, Mario. (2013). The Digital Turn in Architecture 1992 2012. Londres: John Wiley \& Sons.

Deleuze, Gilles y GUATTARI, Félix. (1980). Mil plateaux, Capitalisme et schizophrénie (París: Les Editions de Minuit) Versión en español por José Vázquez Pérez y Umbelina Larraceleta, Mil Mesetas, Capitalismo y esquizofrenia. Buenos Aires: Pre-textos.

Eisenman, Peter. (1991). "Folding in time, the singularity of Rebstock"

Foucault. Michel. (1984). "El juego de Michel Foucault", en Saber y verdad, pp 127-162. Madrid: La Piqueta.

Kipnis, Jeffrey. (2013). A question of qualities. Massachusetts: The Mit Press

Kwinter, Sanford. (2006). "The Judo of Cold Combustion" en REISER, Jesse. 2006. Atlas of Novel Tectonics. Nueva York: Princeton Architectural Press.

Morin, Edgar. (1994). Introducción al pensamiento complejo. Barcelona: Gedisa.

Najle, Ciro. (2004). Machinic Manifesto. Quaderns d'arquitectura i urbanisme. Catalunia: Collegi d'Arquitectes de Catalunya.

Reiser, Jesse. (2006). Atlas of Novel Tectonics. Nueva York: Princeton Architectural Press.

Sarquis, Jorge. (2007). Itinerarios de proyecto 1 y 2, 1 ra ed. Buenos Aires: Nobuko. 